\title{
Influence of depolarization field on polarization states in epitaxial ferroelectric thin films with nonequally biaxial misfit strains
}

\author{
Jie Wang and Tong-Yi Zhang* \\ Department of Mechanical Engineering, Hong Kong University of Science and Technology, Clear Water Bay, Kowloon, \\ Hong Kong, China
}

(Received 14 March 2007; revised manuscript received 2 October 2007; published 22 January 2008)

\begin{abstract}
Depolarization field and misfit strain are two important factors that greatly influence the properties of ferroelectric thin films. The present work studies the effect of depolarization field on polarization states in a single-domain ferroelectric thin film with nonequally biaxial in-plane misfit strains. Three coupled EulerLagrange equations are derived from the minimization of the total free energy and solved numerically. Misfitstrain-misfit-strain phase diagrams are constructed to represent the polarization states. Under the short-circuit boundary condition, the depolarization field shifts the phase boundaries in the phase diagrams, leading to the contraction and expansion of the regions of out-of-plane and in-plane phases, respectively. Under the opencircuit boundary condition, there exists an out-of-plane electretlike state under high compressive misfit strains when a surface polarization $P_{m}$ is included.
\end{abstract}

DOI: 10.1103/PhysRevB.77.014104

\section{INTRODUCTION}

With the remarkable progress in the epitaxial growth of thin films, ferroelectric thin films have found wide applications in new generations of dynamic random access memories, nonvolatile memories, tunable microwave devices, and other microelectronic and electro-optic devices. ${ }^{1-5}$ An epitaxial ferroelectric thin film may have different material properties from its bulk counterpart due to the existence of a stronger depolarization field and lattice mismatch between the film and its substrate. For example, the depolarization field may suppress the ferroelectric property in ultrathin films and change the phase transition order. ${ }^{6,7}$ Mismatch strains between a ferroelectric thin film and its substrate can alter the equilibrium polarization states, shift the phase transition temperature, change the phase transition order, ${ }^{8}$ induce an additional surface polarization, and generate an internal electric field. ${ }^{9}$

A depolarization field is induced by uncompensated charges on the surface and interface of a ferroelectric thin film and/or by inhomogeneous polarization distribution in the film. Under the open-circuit boundary condition, surface charges are not compensated at all, while under the shortcircuit boundary condition, the surface charges are completely compensated so that the depolarization field will be generated only by inhomogeneously distributed polarizations. The inhomogeneity of polarizations is attributed to the so-called intrinsic surface effect of thin films, which can be expressed in terms of the extrapolation length in the phenomenological Landau-Devonshire theory. ${ }^{10,11}$ The extrapolation length describes the distance from the surface of a thin film, at which the polarization will become zero. To describe an inhomogeneous polarization field, the polarization gradient energy should be included in the total free energy of a thin film. An equilibrium state of polarization can be determined by solving Euler-Lagrange equations, which are derived from minimizing the total free energy. Since analytical solutions to the Euler-Lagrange equations are barely possible even in one-dimensional cases, numerical calculations have been performed to obtain the results for most cases. ${ }^{12-14}$
PACS number(s): 77.80.Bh, 77.84.Dy, 77.65.Ly, 77.80.Dj

For simplicity, most studies of ferroelectric thin films based on the Landau-Devonshire theory involve only one polarization component, which is perpendicular ${ }^{9,12,15-17}$ or paralle $1^{13}$ to the surface of a thin film. The uniaxial polarization simplification is not able to describe three-dimensional polarization states in thin films. In the literature, there are some works studying three-dimensional polarization phase diagrams of ferroelectric thin films without considering the depolarization field. ${ }^{14,18,19}$ Recently, Glinchuk et al. ${ }^{20}$ comprehensively studied three-dimensional polarization phase diagrams of ferroelectric thin films with the consideration of depolarization field. In addition, they introduced an additional surface polarization, which was induced by surface piezoelectricity. However, only the short-circuit boundary condition and equally biaxial misfit strains were considered in their study. As mentioned above, charges on the film surface induce a high depolarization field under the open-circuit boundary condition and thus alter the equilibrium polarization states in the ferroelectric film. Nonequally biaxial (or anisotropic) and equally biaxial (or isotropic) misfit strains cause different equilibrium polarization states. $^{19}$ In the present work, we study polarization states in ferroelectric thin films with nonequally biaxial misfit strains under the open-circuit boundary condition and the short-circuit boundary condition.

Figure 1 schematically shows the studied system of a single-domain ferroelectric film epitaxially grown on a thick tetragonal substrate. The film/substrate interface is coherent. The nonequally biaxial misfit strains are induced by the lattice mismatch such that $\varepsilon_{11}=\left(b-a_{0}\right) / b$ and $\varepsilon_{22}=\left(c-a_{0}\right) / c$, and other components of misfit strains are zero, where $b$ and $c$ are the substrate lattice constants and $a_{0}$ is the cubic lattice constant of the film at the freestanding paraelectric state. When the thickness of the substrate is sufficiently larger than the film thickness, the two nonequally biaxial misfit strains will produce a two-dimensional sustained clamping condition for the film. For simplicity, we do not consider any stress-relief mechanisms in the present study such that misfit strains are independent of the film thickness. The rectangular Cartesian system is set up with the $x_{3}$ axis perpendicular to 


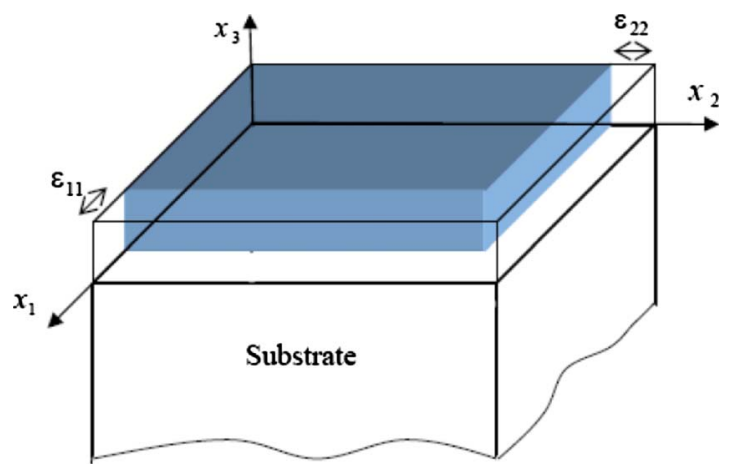

FIG. 1. (Color online) Schematic illustration of nonequally biaxial misfit strains of $\varepsilon_{11}$ and $\varepsilon_{22}$ in a single-domain ferroelectric thin film coherently deposited on an infinitely thick substrate.

the film/substrate interface, as illustrated in Fig. 1.

\section{HELMHOLTZ FREE ENERGY AND EULER-LAGRANGE EQUATIONS}

The Helmholtz free energy density of a ferroelectric thin film with homogeneous polarizations grown on a tetragonal substrate is given in Refs. 8 and 19, which is obtained through Legendre transformation of the standard LandauDevonshire energy. In the present study, we assume that polarizations vary only along the film thickness direction such that the average free energy is calculated from

$$
\tilde{F}=\frac{1}{d} \int_{0}^{d}\left\{f_{L D}\left(P_{i}, \varepsilon_{i j}\right)+f_{G}\left(P_{i, j}\right)+f_{d e p}\left(P_{i}\right)\right\} d x_{3},
$$

where $d$ is the film thickness, $P_{i}$ denotes the $i$ th component of polarization, which is a function of $x_{3}$ only, and $f_{L D}, f_{G}$, and $f_{\text {dep }}$ are the Helmholtz free energy density, the polarization gradient energy, and the depolarization energy density, respectively. The Helmholtz free energy density takes the form of

$$
\begin{aligned}
f_{L D}= & \alpha_{1}^{*} P_{1}^{2}\left(x_{3}\right)+\alpha_{2}^{*} P_{2}^{2}\left(x_{3}\right)+\alpha_{3}^{*} P_{3}^{2}\left(x_{3}\right)+\alpha_{11}^{*}\left[P_{1}^{4}\left(x_{3}\right)+P_{2}^{4}\left(x_{3}\right)\right] \\
& +\alpha_{33}^{*} P_{3}^{4}\left(x_{3}\right)+\alpha_{12}^{*} P_{1}^{2}\left(x_{3}\right) P_{2}^{2}\left(x_{3}\right)+\alpha_{13}^{*}\left[P_{2}^{2}\left(x_{3}\right) P_{3}^{2}\left(x_{3}\right)\right. \\
& \left.+P_{1}^{2}\left(x_{3}\right) P_{3}^{2}\left(x_{3}\right)\right]+\alpha_{111}\left[P_{1}^{6}\left(x_{3}\right)+P_{2}^{6}\left(x_{3}\right)+P_{3}^{6}\left(x_{3}\right)\right] \\
& +\alpha_{112}\left\{P_{1}^{4}\left(x_{3}\right)\left[P_{2}^{2}\left(x_{3}\right)+P_{3}^{2}\left(x_{3}\right)\right]+P_{2}^{4}\left(x_{3}\right)\left[P_{1}^{2}\left(x_{3}\right)\right.\right. \\
& \left.\left.+P_{3}^{2}\left(x_{3}\right)\right]+P_{3}^{4}\left(x_{3}\right)\left[P_{1}^{2}\left(x_{3}\right)+P_{2}^{2}\left(x_{3}\right)\right]\right\} \\
& +\alpha_{123} P_{1}^{2}\left(x_{3}\right) P_{2}^{2}\left(x_{3}\right) P_{3}^{2}\left(x_{3}\right)+\frac{s_{11}\left(\varepsilon_{11}^{2}+\varepsilon_{22}^{2}\right)-2 s_{12} \varepsilon_{11} \varepsilon_{22}}{2\left(s_{11}^{2}-s_{12}^{2}\right)},
\end{aligned}
$$

in which the renormalized coefficients $\alpha_{i j}^{*}$ and $\alpha_{i}^{*}$ are given by

$$
\begin{aligned}
& \alpha_{1}^{*}=\alpha_{1}-\frac{Q_{12}\left(s_{11} \varepsilon_{22}-s_{12} \varepsilon_{11}\right)+Q_{11}\left(s_{11} \varepsilon_{11}-s_{12} \varepsilon_{22}\right)}{s_{11}^{2}-s_{12}^{2}}, \\
& \alpha_{2}^{*}=\alpha_{1}-\frac{Q_{12}\left(s_{11} \varepsilon_{11}-s_{12} \varepsilon_{22}\right)+Q_{11}\left(s_{11} \varepsilon_{22}-s_{12} \varepsilon_{11}\right)}{s_{11}^{2}-s_{12}^{2}},
\end{aligned}
$$

$$
\begin{gathered}
\alpha_{3}^{*}=\alpha_{1}-\frac{Q_{12}\left(\varepsilon_{11}+\varepsilon_{22}\right)}{s_{11}+s_{12}}, \\
\alpha_{11}^{*}=\alpha_{11}+\frac{1}{2} \frac{1}{s_{11}^{2}-s_{12}^{2}}\left[\left(Q_{11}^{2}+Q_{12}^{2}\right) s_{11}-2 Q_{11} Q_{12} s_{12}\right], \\
\alpha_{33}^{*}=\alpha_{11}+\frac{Q_{12}^{2}}{s_{11}+s_{12}}, \\
\alpha_{12}^{*}=\alpha_{12}-\frac{1}{s_{11}^{2}-s_{12}^{2}}\left[\left(Q_{11}^{2}+Q_{12}^{2}\right) s_{12}-2 Q_{11} Q_{12} s_{11}\right]+\frac{Q_{44}^{2}}{2 s_{44}}, \\
\alpha_{13}^{*}=\alpha_{12}+\frac{Q_{12}\left(Q_{11}+Q_{12}\right)}{s_{11}+s_{12}} .
\end{gathered}
$$

For a thin ferroelectric film, the polarization gradient energy density is reduced from the full gradient energy expression used in phase field models ${ }^{21-24}$ and given by

$$
\begin{aligned}
f_{G}= & \frac{1}{2} G_{11}\left(\frac{\partial P_{3}\left(x_{3}\right)}{\partial x_{3}}\right)^{2} \\
& +\frac{1}{2}\left(G_{44}+G_{44}^{\prime}\right)\left[\left(\frac{\partial P_{2}\left(x_{3}\right)}{\partial x_{3}}\right)^{2}+\left(\frac{\partial P_{1}\left(x_{3}\right)}{\partial x_{3}}\right)^{2}\right] .
\end{aligned}
$$

Only the polarization component perpendicular to the plane of thin film, $P_{3}$, can induce depolarization field. Thus, the depolarization field can be derived from Maxwell's equations $^{9,15}$ and given by $E_{d, 3}=-\frac{1}{\varepsilon_{0}}\left[P_{3}\left(x_{3}\right)-\alpha \bar{P}_{3}\right]$ with the thickness-averaged polarization $\bar{P}_{3}=\frac{1}{d} \int_{0}^{d} P_{3}\left(x_{3}\right) d x_{3}$, in which $\alpha$ is introduced to describe compensation of surface charge. For the short-circuit boundary condition, $\alpha=1$ and the surface charges are compensated completely. For the opencircuit boundary condition, $\alpha=0$ and there is no compensation to surface charges at all. The depolarization energy density is then expressed by

$$
f_{\text {dep }}=\frac{1}{2 \varepsilon_{0}}\left[P_{3}\left(x_{3}\right)-\alpha \bar{P}_{3}\right] P_{3}\left(x_{3}\right) .
$$

In Eqs. (2)-(4), $\alpha_{1}=\left(T-T_{0}\right) / 2 \varepsilon_{0} C_{0}$ is the dielectric stiffness, $\alpha_{i j}$ and $\alpha_{i j k}$ are higher-order dielectric stiffnesses, $\varepsilon_{0}=8.85$ $\times 10^{-12} \mathrm{Fm}^{-1}$ is the dielectric constant of vacuum, $T$ and $T_{0}$ denote temperature and the Curie-Weiss temperature, respectively, $C_{0}$ is the Curie constant, $s_{i j}$ are the elastic compliance coefficients, $Q_{i j}$ are electrostrictive constants, and $G_{11}, G_{44}$, and $G_{44}^{\prime}$ are gradient coefficients. In calculations of phase diagrams, $G_{11}=3.46 \times 10^{-10} \mathrm{~m}^{4} \mathrm{~N} / \mathrm{C}^{2}$ and $G_{44}=G_{44}^{\prime}=1.73$ $\times 10^{-10} \mathrm{~m}^{4} \mathrm{~N} / \mathrm{C}^{2}$, and other parameters for $\mathrm{PbTiO}_{3}$ films are tabulated in Table I. ${ }^{8,21}$ The value of the piezoelectric coefficient determines the level of the additional surface polarization, which is set to be $e_{31}=-26 Q / \mathrm{m}^{2}$ as used in Ref. 20 . Note that the present thermodynamic model is able to predict the distribution of inhomogeneous polarizations in the thickness direction. If polarizations are homogeneous in the thickness direction, the gradient energy and the depolarization field will vanish and Eq. (1) will be reduced to the Helmholtz free energy in Ref. 19. 
TABLE I. The parameters used in the calculations for $\mathrm{PbTiO}_{3}$ thin film $\left(\alpha_{1}, \alpha_{i j}, \alpha_{i j k}, Q_{i j}, s_{i j}\right.$, and $T$ are in units $\mathrm{C}^{-2} \mathrm{~m}^{2} \mathrm{~N}, \mathrm{C}^{-4} \mathrm{~m}^{6} \mathrm{~N}$, $\mathrm{C}^{-6} \mathrm{~m}^{10} \mathrm{~N}, \mathrm{C}^{-2} \mathrm{~m}^{4}, \mathrm{~m}^{2} \mathrm{~N}^{-1}$, and ${ }^{\circ} \mathrm{C}$, respectively) (Ref. 8).

\begin{tabular}{lccccccccccc}
\hline \hline $\begin{array}{l}\alpha_{1} \\
\left(\times 10^{5}\right)\end{array}$ & $\begin{array}{c}\alpha_{11} \\
\left(\times 10^{6}\right)\end{array}$ & $\begin{array}{c}\alpha_{12} \\
\left(\times 10^{8}\right)\end{array}$ & $\begin{array}{c}\alpha_{111} \\
\left(\times 10^{8}\right)\end{array}$ & $\begin{array}{c}\alpha_{112} \\
\left(\times 10^{8}\right)\end{array}$ & $\begin{array}{c}\alpha_{123} \\
\left(\times 10^{9}\right)\end{array}$ & $Q_{11}$ & $Q_{12}$ & $Q_{44}$ & $\begin{array}{c}s_{11} \\
\left(\times 10^{-12}\right)\end{array}$ & $\begin{array}{c}s_{12} \\
\left(\times 10^{-12}\right)\end{array}$ & $\begin{array}{c}s_{44} \\
\left(\times 10^{-12}\right)\end{array}$ \\
\hline $3.8(T-479)$ & -73 & 7.5 & 2.6 & 6.1 & -3.7 & 0.089 & -0.026 & 0.0675 & 8.0 & -2.5 & 9.0 \\
\hline \hline
\end{tabular}

The equilibrium polarization distribution is determined by minimizing the total free energy of Eq. (1). Taking the functional variation of Eq. (1) with respect to polarization components $P_{1}\left(x_{3}\right), P_{2}\left(x_{3}\right)$, and $P_{3}\left(x_{3}\right)$ yields the following three coupled nonlinear Euler-Lagrange equations:

$$
\begin{aligned}
0= & 2 \alpha_{1}^{*} P_{1}+4 \alpha_{11}^{*} P_{1}^{3}+2 \alpha_{12}^{*} P_{1} P_{2}^{2}+2 \alpha_{13}^{*} P_{1} P_{3}^{2}+6 \alpha_{111} P_{1}^{5} \\
& +\alpha_{112}\left[4 P_{1}^{3}\left(P_{2}^{2}+P_{3}^{2}\right)+2 P_{2}^{4} P_{1}+2 P_{3}^{4} P_{1}\right]+2 \alpha_{123} P_{1} P_{2}^{2} P_{3}^{2} \\
& -\left(G_{44}+G_{44}^{\prime}\right) P_{1,33}, \\
0= & 2 \alpha_{2}^{*} P_{2}+4 \alpha_{11}^{*} P_{2}^{3}+2 \alpha_{12}^{*} P_{2} P_{1}^{2}+2 \alpha_{13}^{*} P_{2} P_{3}^{2}+6 \alpha_{111} P_{2}^{5} \\
& +\alpha_{112}\left[4 P_{2}^{3}\left(P_{1}^{2}+P_{3}^{2}\right)+2 P_{1}^{4} P_{2}+2 P_{3}^{4} P_{2}\right]+2 \alpha_{123} P_{2} P_{1}^{2} P_{3}^{2} \\
& -\left(G_{44}+G_{44}^{\prime}\right) P_{2,33}, \\
0= & 2 \alpha_{3}^{*} P_{3}+4 \alpha_{33}^{*} P_{3}^{3}+2 \alpha_{13}^{*} P_{3}\left(P_{1}^{2}+P_{2}^{2}\right)+6 \alpha_{111} P_{3}^{5} \\
& +\alpha_{112}\left[4 P_{3}^{3}\left(P_{1}^{2}+P_{2}^{2}\right)+2 P_{1}^{4} P_{3}+2 P_{2}^{4} P_{3}\right]+2 \alpha_{123} P_{3} P_{1}^{2} P_{2}^{2} \\
& -G_{11} P_{3,33}+\frac{P_{3}}{\varepsilon_{0}}-\frac{\alpha}{d \varepsilon_{0}} \int_{0}^{d} P_{3}\left(x_{3}\right) d x_{3},
\end{aligned}
$$

with boundary conditions of

$$
\begin{gathered}
\left.\left(\frac{\partial P_{i}}{\partial x_{3}} \pm \frac{P_{i}}{\delta_{1}}\right)\right|_{x_{3}=0, d}=0 \quad(i=1,2) \\
\left.\left(\frac{\partial P_{3}}{\partial x_{3}} \pm \frac{P_{3}+P_{m}}{\delta_{3}}\right)\right|_{x_{3}=0, d}=0
\end{gathered}
$$

In Eq. (6d), $P_{m}=e_{31} \frac{\varepsilon_{11}+\varepsilon_{22}}{2}$ is the additional surface polarization due to the surface piezoelectric effect caused by broken symmetry on the film surface, ${ }^{20} \delta_{1}$ and $\delta_{3}$ denote the in-plane and out-of-plane extrapolation lengths, respectively, $P_{i}$ denotes $P_{i}\left(x_{3}\right)$, and $P_{i, 33}$ denotes the second derivative of the $i$ th component of the polarization with respect to the coordinate $x_{3}$. We employ finite difference with 100 discrete elements along the thickness to solve the three coupled nonlinear Euler-Lagrange equations numerically. For simplicity, we study only an 8-nm-thick $\mathrm{PbTiO}_{3}$ thin film to explore the effects of depolarization field and misfit strains on the polarization structure at room temperature.

\section{MISFIT-STRAIN-MISFIT-STRAIN PHASE DIAGRAMS OF EPITAXIAL $\mathrm{PbTiO}_{3}$ THIN FILMS}

\section{A. Under the short-circuit boundary condition $(\alpha=1)$}

The thickness-averaged polarizations of $\overline{P_{i}}=\frac{1}{d} \int_{0}^{d} P_{i}\left(x_{3}\right) d x_{3}$ are used to construct misfit-strain-misfit-strain phase dia- grams. We introduce the following notation for different equilibrium phases, which may exist in the film: (i) the $a_{1}$ phase, where $\overline{P_{1}} \neq 0$ and $\overline{P_{2}}=\overline{P_{3}}=0$; (ii) the $a_{2}$ phase, where $\underline{P_{2}} \neq 0$ and $\overline{P_{1}}=\overline{P_{3}}=0$; (iii) the $c$ phase, where $\overline{P_{3}} \neq 0$ and $\overrightarrow{P_{1}}=\overline{P_{2}}=0$; (iv) the $a_{1} c$ phase, where $\overline{P_{3}} \neq 0, \overrightarrow{P_{1}} \neq 0$, and $\overline{P_{2}}=0 ;(v)$ the $a_{2} c$ phase, where $\overline{P_{3}} \neq 0, \overline{P_{2}} \neq 0$, and $\overline{P_{1}}=0$; (vi) the $a_{1} a_{2}$ phase, where $\overline{P_{1}} \neq 0, \overrightarrow{P_{2}} \neq 0$, and $\overline{P_{3}}=0$, and the aa phase when $\overline{P_{1}}=\overline{P_{2}}$ in this case; (vii) the $r$ phase, where $\overline{P_{3}} \neq 0, \overline{P_{1}} \neq 0$, and $\overline{P_{2}} \neq 0$. Figure 2 shows the misfit-strainmisfit-strain phase diagrams under the short-circuit boundary condition with $\delta_{1}=\delta_{3}=3 \mathrm{~nm},{ }^{25}$ where the solid and dotdashed lines denote the phase diagrams with and without the depolarization field of Eq. (5), respectively. One monoclinic $r$ phase, two orthorhombic $a_{1} c$ and $a_{2} c$ phases, and one tetragonal $c$ phase show up in the phase diagrams with and without the depolarization field. The phase diagram with the depolarization field looks similar to that without the depolarization field. But the depolarization field makes the phase boundaries shift such that the region of the out-of-plane phase is reduced.

In general, the values of extrapolation lengths, $\delta_{1}$ and $\delta_{3}$, should be different. Different values between $\delta_{1}$ and $\delta_{3}$ are also employed to study the effect of extrapolation length on polarization state. For example, we use $\delta_{1}=3 \mathrm{~nm}$ and $\delta_{3}$ $=4 \mathrm{~nm}$ in the calculations and plot in Fig. 3 a similar misfitstrain-misfit-strain phase diagram under the short-circuit boundary condition. Compared to the phase diagrams in Fig. 2 , the region of $c$ phase and $r$ phase is reduced in the phase diagrams of Fig. 3. If $\delta_{1}=4 \mathrm{~nm}$ and $\delta_{3}=3 \mathrm{~nm}$ are used, there is only a $c$ phase in the entire strain region. These results show that extrapolation lengths have an important influence

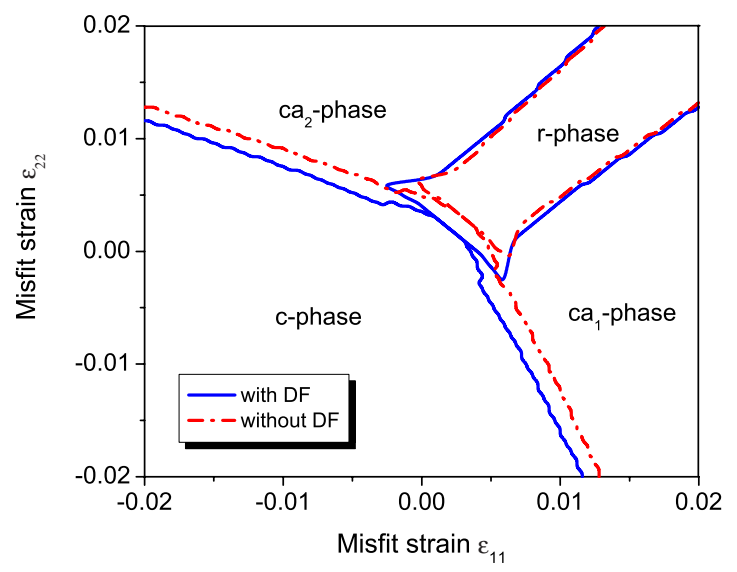

FIG. 2. (Color online) Misfit-strain-misfit-strain phase diagrams for the 8-nm-thick $\mathrm{PbTiO}_{3}$ thin film under the short-circuit boundary condition with and without depolarization field (DF) at room temperature, where $\delta_{1}=\delta_{3}=3 \mathrm{~nm}$. 


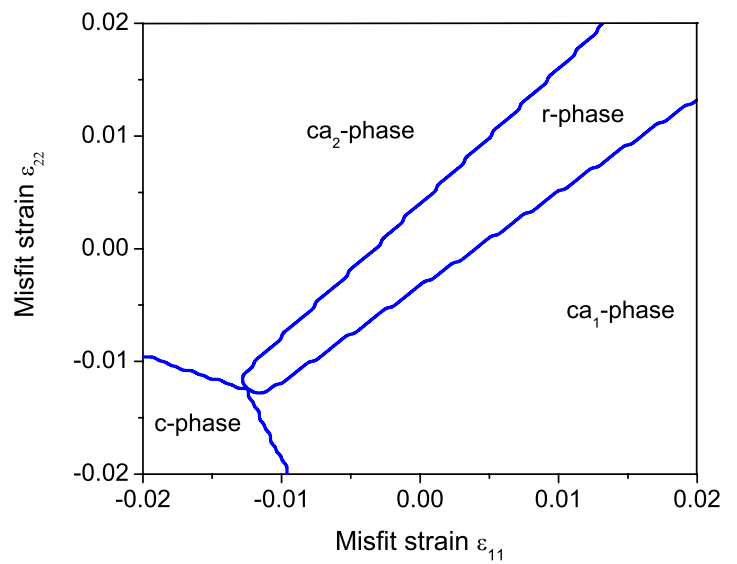

FIG. 3. (Color online) Misfit-strain-misfit-strain phase diagram for the 8-nm-thick $\mathrm{PbTiO}_{3}$ thin film under the short-circuit boundary condition at room temperature with extrapolation lengths of $\delta_{1}=3 \mathrm{~nm}$ and $\delta_{3}=4 \mathrm{~nm}$.

on the equilibrium polarization states in ferroelectric thin films. The value of $\delta$ should be determined by first-principles calculations and/or by carefully designed experiments, which will be the future task of research. To focus on the investigation of the depolarization field effect, the same value of $\delta_{1}=\delta_{3}=3 \mathrm{~nm}$ is used hereafter. ${ }^{25}$

Figure 4 shows the misfit-strain-misfit-strain phase diagrams under the short-circuit boundary condition when the additional surface polarization $P_{m}$ is ignored, where again the solid and dot-dashed lines denote the phase diagrams with and without the depolarization field, respectively. Comparing Fig. 4 with Fig. 2 indicates that out-of-plane polarizations disappear in some regions of monoclinic $r$ phase and orthorhombic $a_{1} c$ and $a_{2} c$ phases, which makes pure in-plane $a_{1}, a_{2}$, and $a_{1} a_{2}$ phases appear in the phase diagrams of Fig. 4. This result shows that, because of nonzero additional surface polarization, the out-of-plane component of polarization exists in the entire region of the phase diagrams. Figure 4

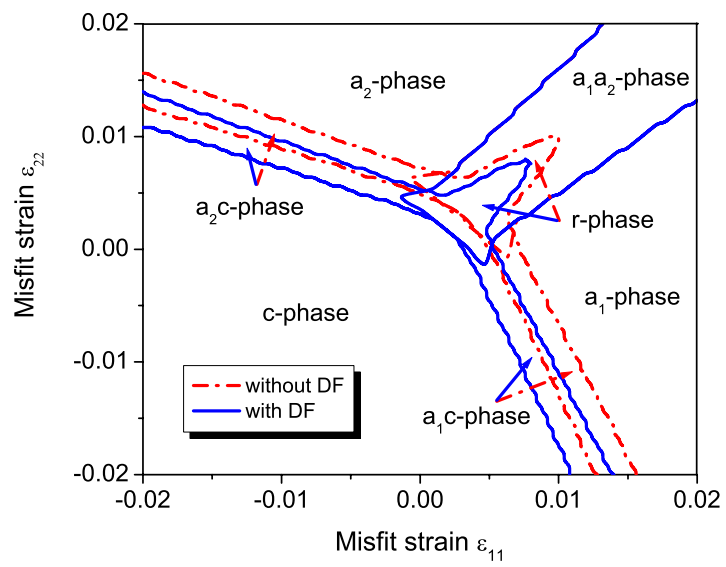

FIG. 4. (Color online) Misfit-strain-misfit-strain phase diagrams for the 8-nm-thick $\mathrm{PbTiO}_{3}$ thin film under the short-circuit boundary condition with and without depolarization field (DF) at room temperature when the additional surface polarization is ignored and $\delta_{1}=\delta_{3}=3 \mathrm{~nm}$.

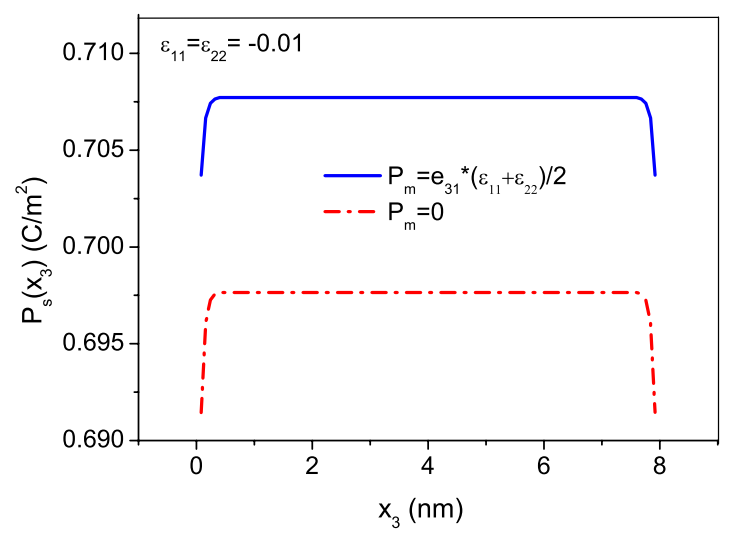

FIG. 5. (Color online) Profiles of the polarization magnitude along the thickness direction in the 8-nm-thick $\mathrm{PbTiO}_{3}$ thin film under the short-circuit boundary condition at room temperature with and without the additional surface polarization, where $\delta_{1}=\delta_{3}$ $=3 \mathrm{~nm}$.

illustrates that the depolarization field makes the phase boundaries shift such that the region of the out-of-plane phase is reduced and the region of the in-plane phases is extended. This is because the depolarization field is only dependent on the out-of-plane polarization component. For the same reason, the phase boundaries between in-plane phases are independent of the depolarization field. That is why the phase boundary between the $a_{1}$ and $a_{1} a_{2}$ phases and the phase boundary between the $a_{2}$ and $a_{1} a_{2}$ phases are not shifted when the depolarization field is considered, as shown on the right-upper part of Fig. 4. To investigate the effect of the additional surface polarization, Fig. 5 gives the profile of polarization magnitude, $P_{s}=\sqrt{P_{1}^{2}+P_{2}^{2}+P_{3}^{2}}$, along the film thickness direction with and without the additional surface polarization under the short-circuit boundary condition, where $\varepsilon_{11}=\varepsilon_{22}=-0.01$. It is found that the additional surface polarization enhances the magnitude of polarization.

\section{B. Under the open-circuit boundary condition $(\alpha=0)$}

Figure 6 shows the misfit-strain-misfit-strain phase diagram under the open-circuit boundary condition. When the additional surface polarization is considered, three ferroelectric phases-namely, one monoclinic $r$ phase and two orthorhombic phases $c a_{1}$ and $c a_{2}$-appear within different ranges of misfit strains. The monoclinic $r$ phase only exists around the equally axial misfit strains under both compression and tension, while two orthorhombic phases $c a_{1}$ and $c a_{2}$ exist in the region of misfit strains with higher anisotropy. It is interesting that there is an electretlike state under large compressive misfit strains, which is shown in the lower-left corner in the phase diagram. The electretlike polar state is basically the $c$ phase, and the additional surface polarization makes it polarized. The electretlike state has a much smaller polarization due to the depolarization field, which can be found from the inset of Fig. 9(a). Figure 6 shows that the compressive misfit strains can induce transition from the electretlike state to the ferroelectric state and this transition is thickness dependent. ${ }^{20}$ 


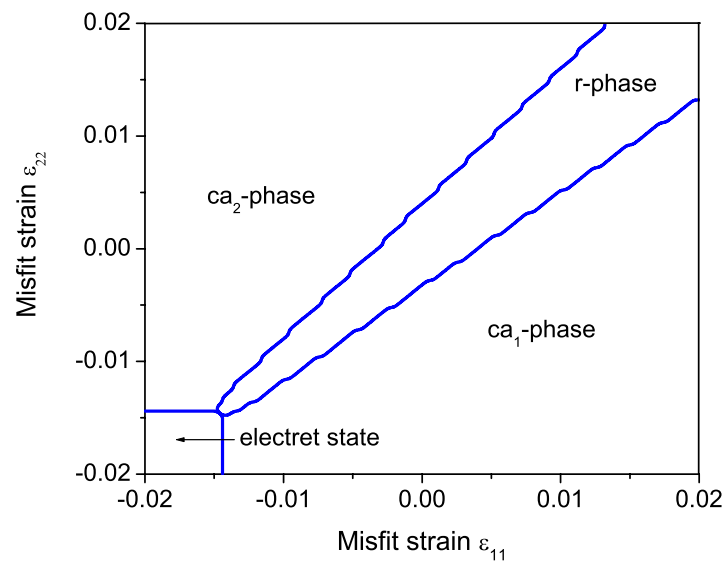

FIG. 6. (Color online) Misfit-strain-misfit-strain phase diagram for the 8-nm-thick $\mathrm{PbTiO}_{3}$ thin film under the open-circuit boundary condition at room temperature, where $\delta_{1}=\delta_{3}=3 \mathrm{~nm}$.

If the additional surface polarization $P_{m}$ is ignored, the misfit-strain-misfit-strain phase diagram in Fig. 6 will be changed to that shown in Fig. 7. The phase diagram in Fig. 7 has the same shape as that in Fig. 6. But there is no out-ofplane component of polarization in Fig. 7, meaning that the monoclinic $r$ phase, the orthorhombic phases $c a_{1}$ and $c a_{2}$, and electretlike states in Fig. 6 are replaced by the orthorhombic phase $a_{1} a_{2}$, tetragonal phases $a_{1}$ and $a_{2}$, and paraelectric phase in Fig. 7, respectively. The results indicate that, because there is no surface charge compensation under the open-circuit boundary condition, the depolarization fields completely suppress the ferroelectric properties in the film with large biaxial compressive misfit strains.

\section{Thin films with partial surface charge compensation}

In order to demonstrate the competition between depolarization effects and strain effects, thin films with partial surface charge compensation are studied by using different values of $\alpha$ (other than 0 or 1). It is found that the phase

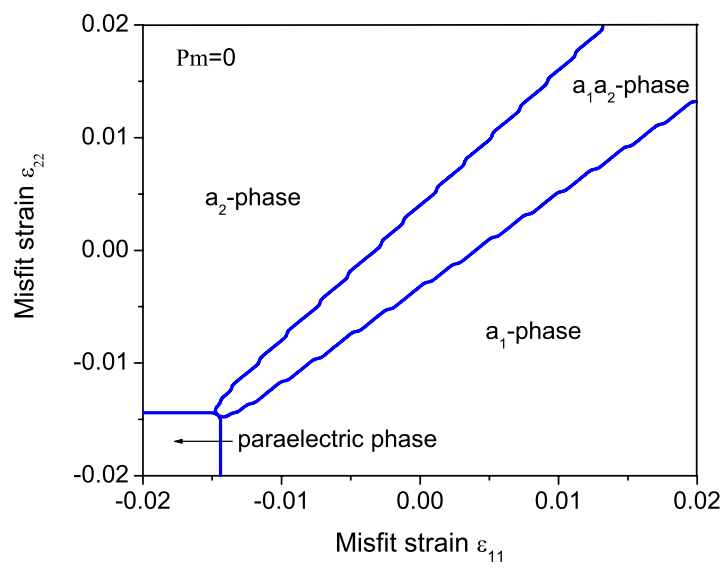

FIG. 7. (Color online) Misfit-strain-misfit-strain phase diagram for the 8-nm-thick $\mathrm{PbTiO}_{3}$ thin film under the open-circuit boundary condition at room temperature when the additional surface polarization $P_{m}$ is ignored, where $\delta_{1}=\delta_{3}=3 \mathrm{~nm}$.

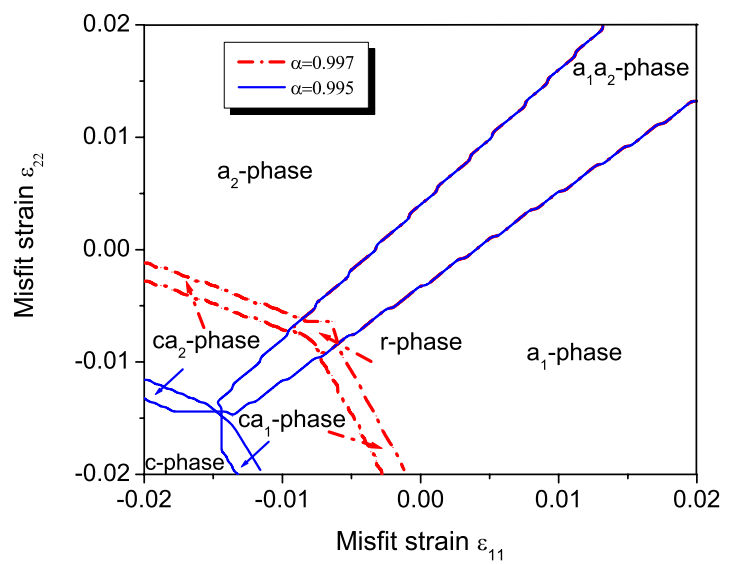

FIG. 8. (Color online) Misfit-strain-misfit-strain phase diagram for the 8-nm-thick $\mathrm{PbTiO}_{3}$ thin films with partial surface charge compensation at room temperature when the additional surface polarization $P_{m}$ is ignored, where $\delta_{1}=\delta_{3}=3 \mathrm{~nm}$.

diagram remains unchanged when $\alpha$ increases from 0 to 0.99 , while the phase diagram changes gradually from that of the open-circuit case $(\alpha=0)$ to that of the short-circuit case $(\alpha=1)$ when $\alpha$ increases from 0.99 to 1 . Figure 8 shows the misfit-strain-misfit-strain phase diagrams for the 8-nm-thick $\mathrm{PbTiO}_{3}$ thin films under partial surface charge compensation at room temperature when $\alpha=0.995$ and $\alpha=0.997$, respectively. The phase diagrams with different $\alpha$ values show that surface charge compensation increases the region of out-ofplane phase through the movement of the phase boundaries between the in-plane phase and out-of-plane phase, but the phase boundaries between different in-plane phases is unchanged. This result is reasonable because more charges at surface with less compensation can induce a larger depolarization field which suppresses the out-of-plane phase in the ferroelectric thin films. The out-of-plane phase is completely suppressed when $\alpha$ is smaller than 0.99 , which can be found from the phase diagram in Fig. 7. When the surface charges are completely compensated $(\alpha=1)$, the region of out-ofplane phase is the largest, as shown in Fig. 4 with the depolarization field, among all cases.

\section{Thickness-averaged polarization and polarization profile}

Figures 9(a) and 9(b) show the dependence of the thickness-averaged polarization components $\overline{P_{3}}$ and $\overline{P_{1}}$ on the misfit strain, respectively, where the solid lines and dotdashed lines show the results under the short-circuit boundary condition and the open-circuit boundary condition, respectively. The inset in Fig. 9(a) is the rescaled plot of $\overline{P_{3}}$ under the open-circuit boundary condition of $\alpha=0$. Without any surface charge compensation, the out-of-plane polarization under the open-circuit boundary condition is much smaller than that under the short-circuit boundary condition, which is attributed to the difference in the depolarization field under the two boundary conditions. A large depolarization field under the open-circuit boundary condition causes the decrease in the out-of-plane polarization, and the result is consistent with other theoretical predictions. ${ }^{26,27}$ In addition, 

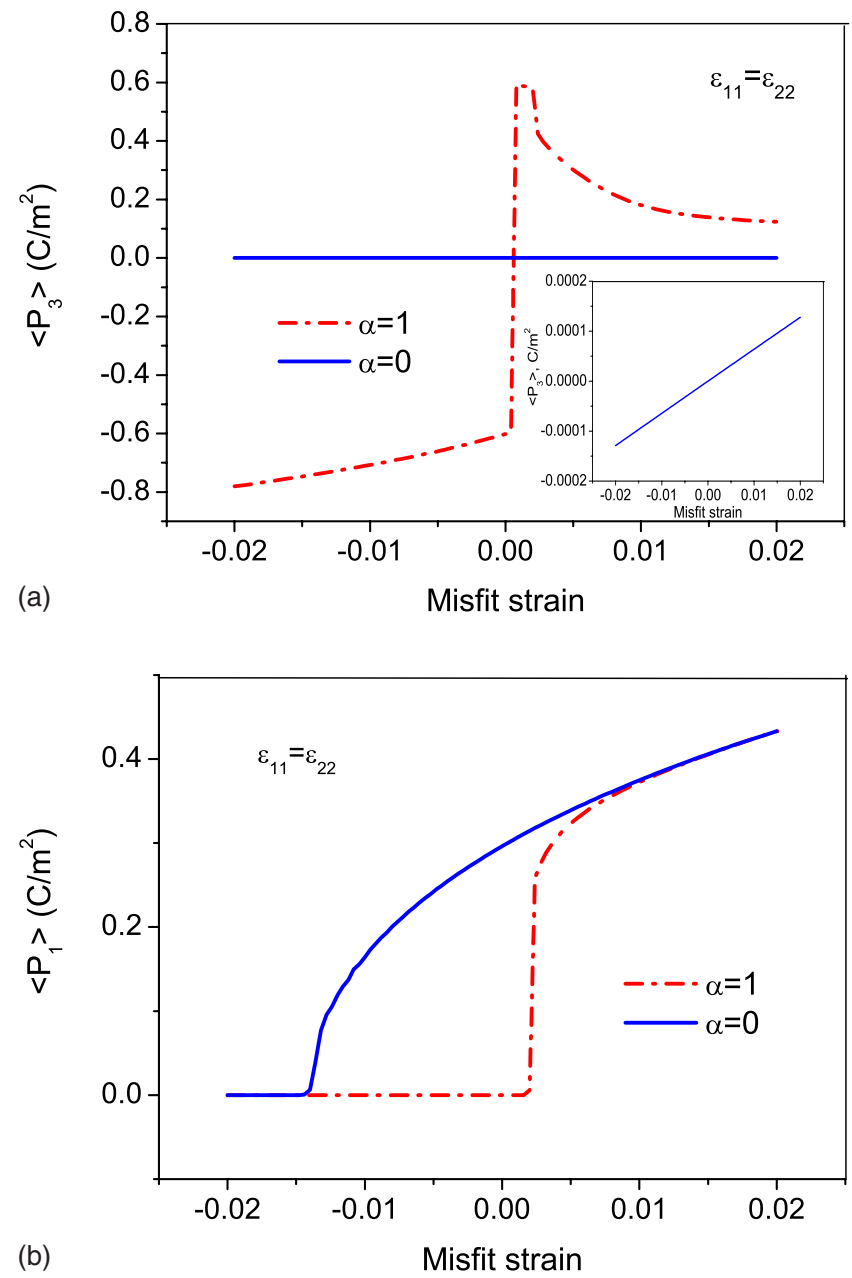

FIG. 9. (Color online) Thickness-averaged polarization components (a) $\overline{P_{3}}$ and (b) $\overline{P_{1}}$ as a function of equally biaxial misfit strain under the short-circuit boundary condition $\alpha=1$ and under the opencircuit boundary condition $\alpha=0$. The inset in (a) is the rescaled plot of $\overline{P_{3}}$ for $\alpha=0$.

the direction of out-of-plane polarization is dependent on the sign of the misfit strains, which is shown in the inset of Fig. 9(a). Figure 9(b) indicates that the in-plane polarizations are also different for the two boundary conditions in the strain range from -0.015 to 0.01 , but they are the same independent of the boundary conditions under larger compressive and tensile strains.

Figures $10(\mathrm{a})$ and $10 \underline{(\mathrm{b})}$ show the profiles of the polarization components $\overline{P_{1}}$ and $\overline{P_{3}}$ along the film thickness direction under the open-circuit boundary condition, respectively. The out-of-plane polarization component approaches zero inside the film, but it becomes larger near the film surface and interface. However, there is an inverse trend for the in-plane polarization component, which is larger inside the film than that near the surface and interface.

\section{CONCLUDING REMARKS}

The present work systematically studies the polarization states of a single-domain thin film grown on a tetragonal
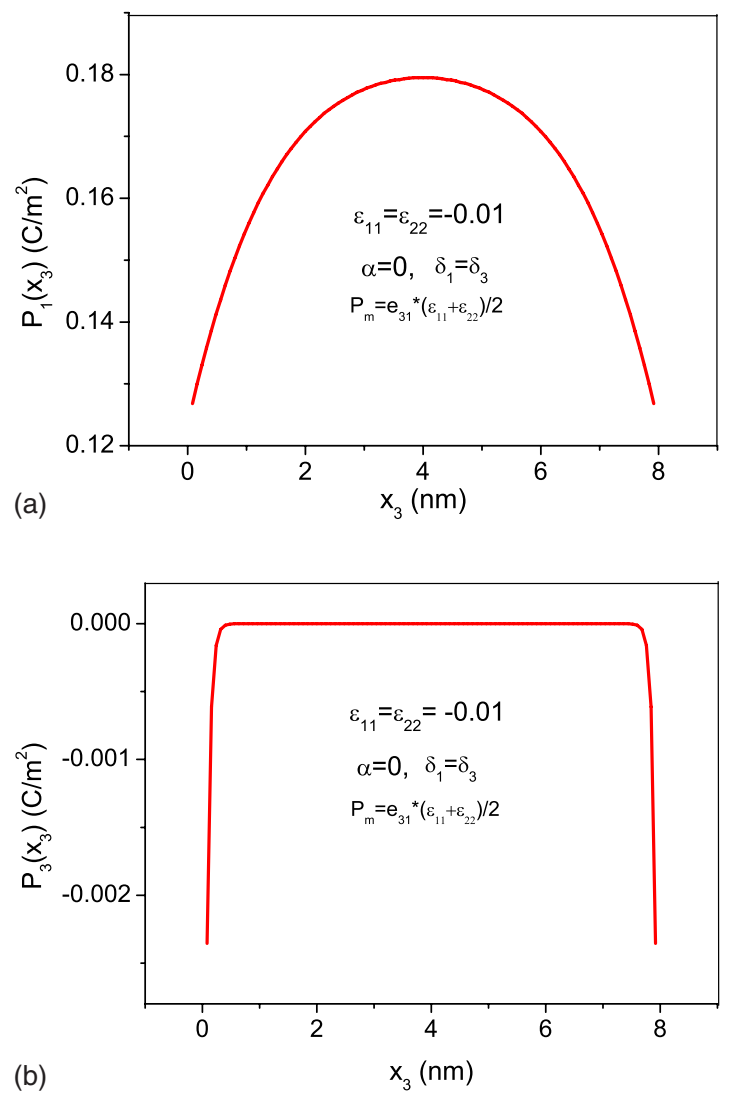

FIG. 10. (Color online) Profiles of polarization components (a) $P_{1}$ and (b) $P_{3}$ along the thickness direction in the 8-nm-thick $\mathrm{PbTiO}_{3}$ thin film under the open-circuit boundary condition at room temperature.

substrate with different boundary conditions. The thermodynamic function expressed by Eqs. (1)-(5) is the theoretical foundation including the depolarization field. Three coupled Euler-Lagrange equations are derived from the variation of the thermodynamic function with the short-circuit and opencircuit boundary conditions. Simultaneously solving the three coupled Euler-Lagrange equations is a minimization process of the thermodynamic function. An 8-nm-thick single-domain $\mathrm{PbTiO}_{3}$ thin film is taken as an example to construct the misfit-strain-misfit-strain phase diagrams at room temperature. The results clearly illustrate the change in the phase boundaries caused by the difference in the boundary conditions. The depolarization field reduces the region of the out-of-plane phase and increases the region of the inplane phases in both misfit-strain-misfit-strain phase diagrams. Moreover, the depolarization field may induce the phase transition from the orthorhombic phases to the inplane tetragonal phases in the ferroelectric thin films with nonequally biaxial misfit strains. This is because in the inplane tetragonal phases there is no depolarization field in the thickness direction of the ferroelectric thin films, meaning that the depolarization field has no effect on the polarization instability of the in-plane tetragonal phases.

It is should be noted that only single-domain films are investigated in the present study. Real ferroelectric thin films may form multidomain structures with $180^{\circ}$ and/or $90^{\circ}$ do- 
main walls so that the depolarization field is reduced ${ }^{28}$ However, when the film thickness is sufficiently small, the singledomain structure will be predominent, ${ }^{23}$ thereby indicating that the results obtained from the present study may be more applicable to extrathin ferroelectric films.

\section{ACKNOWLEDGMENTS}

This work was fully supported by an RGC grant from the Research Grants Council of the Hong Kong Special Administrative Region, China.
*Corresponding author. FAX: (852) 2358-1543. mezhangt@ust.hk

${ }^{1}$ M. J. Dalberth, R. E. Stauber, J. C. Price, C. T. Roger, and D. Galt, Appl. Phys. Lett. 72, 507 (1998).

${ }^{2}$ Q. X. Jia, A. T. Findikoglu, D. Reagor, and P. Lu, Appl. Phys. Lett. 73, 897 (1998).

${ }^{3}$ H. N. Lee, D. Hesse, N. Zakharov, and U. Gosele, Science 296, 2006 (2002).

${ }^{4}$ C. H. Ahn, K. M. Rabe, and J. M. Triscone, Science 303, 488 (2004).

${ }^{5}$ C. L. Chen, H. H. Feng, Z. Zhang, A. Brazdeikis, Z. J. Huang, W. K. Chu, C. W. Chu, F. A. Miranda, F. W. Van Keuls, R. R. Romanofsky, and Y. Liou, Appl. Phys. Lett. 75, 412 (1999).

${ }^{6}$ J. Junquera and P. Ghosez, Nature (London) 422, 506 (2003).

${ }^{7}$ I. P. Batra, P. Wurfel, and B. D. Silverman, Phys. Rev. Lett. 30, 384 (1973)

${ }^{8}$ N. A. Pertsev, A. G. Zembilgotov, and A. K. Tagantsev, Phys. Rev. Lett. 80, 1988 (1998).

${ }^{9}$ M. D. Glinchuk and A. N. Morozovska, J. Phys.: Condens. Matter 16, 3517 (2004).

${ }^{10}$ W. L. Zhong, Y. G. Wang, P. L. Zhang, and B. D. Qu, Phys. Rev. B 50, 698 (1994).

${ }^{11}$ S. Li, J. A. Eastman, Z. Li, C. M. Foster, R. E. Newnham, and L. E. Cross, Phys. Lett. A 212, 341 (1996).

${ }^{12}$ V. C. Lo, J. Appl. Phys. 94, 3353 (2003).

${ }^{13}$ K. H. Chew, C. L. Wang, F. G. Shin, H. L. W. Chan, and D. R. Tilley, Solid State Commun. 123, 457 (2002).
${ }^{14}$ J. Zhang, Z. Yin, and M. S. Zhang, Phys. Lett. A 310, 479 (2003).

${ }^{15}$ R. Kretschmer and K. Binder, Phys. Rev. B 20, 1065 (1979).

${ }^{16}$ Y. G. Wang, W. L. Zhong, and P. L. Zhang, Phys. Rev. B 51, 5311 (1995).

${ }^{17}$ A. G. Zembilgotov, N. A. Pertsev, H. Kohlstedt, and R. Waser, J. Appl. Phys. 91, 2247 (2002).

${ }^{18}$ A. Y. Emelyanov, N. A. Pertsev, and A. L. Kholkin, Phys. Rev. B 66, 214108 (2002).

${ }^{19}$ J. Wang and T. Y. Zhang, Appl. Phys. Lett. 86, 192905 (2005).

${ }^{20}$ M. D. Glinchuk, A. N. Morozovska, and E. A. Eliseev, J. Appl. Phys. 99, 114102 (2006).

${ }^{21}$ Y. L. Li, S. Y. Hu, Z. K. Liu, and L. Q. Chen, Appl. Phys. Lett. 81, 427 (2002).

${ }^{22}$ J. Wang, S. Q. Shi, L. Q. Chen, Y. L. Li, and T. Y. Zhang, Acta Mater. 52, 749 (2004).

${ }^{23}$ J. Wang and T. Y. Zhang, Phys. Rev. B 73, 144107 (2006).

${ }^{24}$ Y. L. Li, S. Y. Hu, Z. K. Liu, and L. Q. Chen, Acta Mater. 50, 395 (2002).

${ }^{25}$ K. Ishikawa and T. Uemori, Phys. Rev. B 60, 11841 (1999).

${ }^{26}$ M. E. Lines and A. M. Glass, Principles and Applications of Ferroelectrics and Related Materials (Clarendon, Oxford, 1977).

${ }^{27}$ T. Lu and W. Cao, Phys. Rev. B 66, 024102 (2002).

${ }^{28}$ W. Y. Shih, W. H. Shih, and I. A. Aksay, Phys. Rev. B 50, 15575 (1994). 